\title{
INVESTIGACIÓN EN TEORÍA DE LA PSICOLOGÍA
}





\title{
Proceso, coherencia, cotidianidad y qualia como desafíos explicativos para la psicología
}

\author{
Process, coherence, everyday life and qualia as explanatory \\ challenges for psychology
}

Jorge Larreamendy-Joerns

Universidad de los Andes

jlarream@uniandes.edu.co

Artículo de reflexión recibido el 12/04/10 y aprobado el 31/05/10

\begin{abstract}
Resumen
En este artículo se someten a discusión cuatro desafíos explicativos de la psicología a nivel teórico: primero, la necesidad de avanzar hacia explicaciones históricas y de proceso; segundo, la necesidad de observar estándares más estrictos de coherencia explicativa; tercero, la urgencia de abordar fenómenos en el ámbito de la cotidianidad; y cuarto, la necesidad de dar cuenta de fenómenos en el orden de la qualia y la experiencia. En primer término se exploran las dificultades para identificar desafíos alrededor de los cuales pueda converger la psicología en su conjunto. Posteriormente, se examinan logros consolidados en la disciplina psicológica como una manera de aproximarse a la identificación de retos teóricos de relevancia general. Finalmente, se dedica una sección a cada uno de los desafíos teóricos anteriormente mencionados. El artículo concluye con un aparte de consideraciones finales.
\end{abstract}

Palabras clave: Qualia, Coherencia explicativa, Explicaciones de proceso, Vida cotidiana, Psicología.

\begin{abstract}
TThis article discusses four explanatory challenges for psychology at a theoretical level: first, the need to move towards historical and process explanations; second, the need to observe strict standards of explanatory coherence; third, the urgency of addressing phenomena in the field of everyday life; and fourth, the need to account for phenomena in the order of qualia and experience. First, the article explores some difficulties in identifying challenges around which psychology as a whole may converge. Subsequently, it examines the achievements consolidated in the psychological discipline as a way to approach the identification of theoretical challenges of general relevance. Finally, a section is devoted to each of the aforementioned theoretical challenges. The article concludes with final considerations.
\end{abstract}

Key words: Qualia, Explanatory coherence, Process explanations, Everyday life, Psychology. 



\section{Introducción}

La primera década del siglo XX fue prodigiosa en términos de sus aportes a la naciente ciencia psicológica. Como se recordará, las dos últimas décadas del siglo XIX fueron, en cierto modo, el tiempo de la institucionalización de la disciplina, en especial con la fundación en 1879 del laboratorio de psicología en Leipzig por parte de Wundt y la publicación en 1890 de Principles of Psychology de James. Con el advenimiento del nuevo siglo, sin embargo, una vez establecida la psicología en la universidad norteamericana y europea, se sentaron las bases para algunas de las más destacadas tradiciones psicológicas del siglo por venir. Algunos hitos relevantes fueron la publicación en 1900 de La interpretación de los sueños de Freud, la edición en 1903 de Educational Psychology de Edward Thorndike, la publicación en 1905 del test de inteligencia de Binet, y la publicación en 1906 de los primeros estudios de Pavlov sobre condicionamiento. En las décadas siguientes veríamos consolidarse el programa del psicoanálisis, el ámbito profesional de la psicología educativa, el campo de la evaluación del desarrollo y la inteligencia, y los modelos basados en el condicionamiento clásico e instrumental. Desde luego, el ímpetu característico de las primeras décadas de la psicología debe entenderse en el trasfondo de una disciplina que, como si estuviera siguiendo la pendiente de una curva de aprendizaje, apenas estaba estableciendo sus paradigmas fundamentales, procediendo, sin embargo, con vigor y celeridad.

Como cabría esperar, un siglo después, la situación no es la misma. Hoy en día, tenemos ya cien ańos de desarrollos empíricos y debates conceptuales. Algunas tradiciones, como es el caso del análisis experimental del comportamiento y, en cierto sentido, la psicología cognitiva de corte computacional han ingresado en las aguas relativamente tranquilas de la ciencia normal, luego de borrascosos comienzos y de revoluciones intermitentes. Avances recientes en estas áreas parecen implicar menos el origen de nuevos paradigmas que el ajuste de los ya existentes. Otras tendencias, como la teoría sociocultural y la psicología discursiva y crítica - para nombrar sólo algunas- han implicado giros significativos y rupturas con respecto a prácticas disciplinares tradicionales. De allí que su estatuto sea frecuentemente contestado y su reconocimiento pleno esté (con todas las implicaciones del caso) aún en disputa.

El panorama de las ciencias también ha cambiado: hace 100 años, la física, luego del renovado impulso de las aportaciones de Maxwell y Einstein sobre electromagnetismo, constituía el modelo a seguir, como en su momento lo proclamaron los filósofos del Círculo de Viena (Packer, 2010). En la actualidad, es la biología, ya no la física, la disciplina que detenta las mayores promesas; y, 
desde luego, la psicología está ubicada hoy en día, aunque de manera polémica para algunos, en el seno de unas ciencias sociales y humanas que, a comienzos del siglo $\mathrm{XX}$ apenas se vislumbraban como disciplinas autónomas, como resultado del pensamiento de Marx, Weber y Durkheim.

¿Qué desafíos le esperan a la psicología en las próximas décadas? O mejor, ¿qué logros disciplinares deberíamos garantizar? Supongo que cualquier intento de anticipar los avances sustantivos de la psicología en las próximas décadas, está de suyo condenado a la insuficiencia, cuando no al fracaso, excepto que se reconozca como exhaustivo y profético. Como sabemos, la profecía es una práctica de muy dudosa reputación en ciencia, pero incluso si resultase válida tendría el indeseable efecto de convertir nuestros próximos cien años en un escenario de aburrimiento anticipado. En este artículo quisiera, no obstante, tomar una diferente ruta y someter a discusión lo que denominaría cuatro desafíos transversales de la psicología a nivel teórico: primero, la necesidad de avanzar hacia explicaciones históricas y de proceso; segundo, la necesidad de observar estándares más estrictos de coherencia explicativa; tercero, la urgencia de abordar fenómenos en el ámbito de la cotidianidad; y cuarto, la necesidad de dar cuenta de fenómenos en el orden de la qualia y la experiencia. Desde luego, no hay razones para suponer que los anteriores desafíos sean los únicos o incluso los más importantes. Pero los desafíos propuestos están relacionados con asuntos que la disciplina en su conjunto debería tratar si aspira a potenciar el valor heurístico de sus explicaciones.

El presente artículo está organizado de la siguiente manera. En primer lugar, se exploran las dificultades para identificar retos o desafíos alrededor de los cuales pueda converger la psicología en su conjunto. Posteriormente, se examinan logros consolidados en la disciplina psicológica como una manera de aproximarse a la identificación de retos teóricos de relevancia general. Finalmente, se dedica una sección a cada uno de los desafíos teóricos anteriormente mencionados. El artículo concluye con un aparte de consideraciones finales.

\section{Obstáculos para la identificación de desafíos sustantivos}

Nuevamente, ¿qué desafíos le esperan a la psicología en las próximas décadas? $Y$, ¿por qué es difícil identificar desafíos de amplia relevancia disciplinaria? En principio, una de las dificultades mayores para precisar retos futuros, con los cuales una amplia audiencia pueda identificarse, radica en la formidable diversidad y, por qué no decirlo, dispersión de la psicología como ámbito de indagación y ejercicio profesional. A manera de ejemplo, Green (2009) ha señalado recientemente cómo, en las últimas décadas, bajo la influencia de modelos computacionales 
y nuevas tecnologías de medición y visualización, la cognición, la percepción y la neuropsicología parecen inclinarse cada vez más hacia las ciencias naturales duras, al punto de muchas veces asumir posturas eliminacionistas. Simultáneamente, sin solución de continuidad, un número creciente de psicólogos desarrolla aproximaciones inspiradas en la filosofía postmoderna, la hermenéutica, el pensamiento crítico y la perspectiva de género (Ibañez \& Íñiguez-Rueda, 1997; Parker, 1999; Sampson, 1981). Muchas de estas aproximaciones han cuestionado la adecuación conceptual de nociones fundacionales de la ciencia psicológica tradicional, como el individuo y la mente, al igual que han criticado, como se sabe, la designación de la psicología como disciplina científica (cuando menos, en su versión positivista). Por supuesto, no se trata de un movimiento pendular entre el eliminacionismo y la hermenéutica, sino de dos proyectos de psicología dispares, cuya integración no creo sea deseable por ninguna de las partes. De hecho, Green se preguntaba si quienes se ubican programáticamente entre estos dos extremos estarán en capacidad de mantener su posición mediadora o si, por el contrario, el campo de la disciplina terminará polarizándose inevitablemente. La polarización tiene como consecuencia que criterios de relevancia se vuelvan criterios locales. Así, lo que resulta un desafío para el programa eliminacionista es un sinsentido para el programa hermenéutico y viceversa.

Es fácil ver, por otra parte, que la diversidad de la psicología como disciplina no se limita, para emplear los términos del filósofo italiano Marcello Pera (1994), a la quaestio juris; abarca también la quaestio facti. Es decir, no solamente están en contención los modelos interpretativos o explicativos de los fenómenos, sino también los parámetros para decidir lo que puede o no ser admitido como evidencia; dicho de otra manera, la naturaleza misma del hecho psicológico.

A lo anterior, debemos agregar la diversidad metodológica derivada de las decisiones o tradiciones asumidas sobre lo que constituye un fenómeno psicológico. Pese los esfuerzos por normalizar protocolos investigativos en psicología, ${ }^{1}$ hoy en día los métodos son más diversos que nunca, incluso en el contexto de campos particulares (las ciencias del aprendizaje [learning sciences] son un ejemplo claro, ver Sawyer, 2006). De allí que diferentes aproximaciones metodológicas, ligadas ellas a concepciones de la evidencia, puedan establecer retos distintos.

Esta situación de diversidad es singularmente distinta de la de disciplinas como la física e incluso la biología que, pese a su amplitud fenoménica, pueden legítimamente especular sobre la existencia de principios fundamentales, como los expuestos por Feynmann (1970) en sus lecciones introductorias a la física; o

1 Piénsese, por ejemplo, en las prescripciones metodológicas típicas de los textos introductorios en psicología o en las indicaciones de diseño implícitas en convocatorias o políticas de financiación. 
de teorías finales, como lo ha sugerido Weinberg (1993); o bien de marcos unificadores, como sucede en la biología con la síntesis neo-darwiniana (Mayr, 1983). No se trata, en mi opinión, de incurrir de nuevo en el defectuoso argumento de imputar a las ciencias naturales una simplicidad interpretativa que no tienen, o de revivir la oposición irreconciliable entre las ciencias físicas y las ciencias cognitivas a la manera de Dilthey. Se trata, sí, de reconocer que la psicología es profundamente heteróclita, al margen de si su diversidad es una consecuencia de su nivel de desarrollo histórico, o por el contrario, una característica inherente al dominio. Como corolario, podríamos decir entonces que el problema de los retos debería, con justicia, ventilarse en el contexto de dominios específicos de indagación, bajo el entendido de que la psicología no es una disciplina unificada, y probablemente tampoco un campo en vías de unificación.

Quisiera mencionar una dificultad adicional, cuando se trata de identificar los retos que esperan a la psicología en las décadas por venir: la duplicidad de la psicología como disciplina y profesión, duplicidad que ha jugado un papel esencial en Colombia (a diferencia de su importancia relativa en América del Norte y Europa). Aunque los nexos entre el saber disciplinario y el quehacer profesional no se reducen a la mera aplicación de principios generales y a la incorporación en esquemas explicativos abstractos de condiciones contingentes, es claro que lo relevante disciplinariamente no tiene por qué serlo profesionalmente y viceversa. Esto es especialmente claro en lo relativo a retos gremiales (si admite la expresión) o a las relaciones entre las profesiones (y los profesionales), que deben distinguirse de las relaciones entre las disciplinas (Becher $\&$ Trowler, 1989). Otro tanto sucede con algunos de los compromisos sociales o políticos que los psicólogos deberíamos asumir y que no tienen porqué ser entendidos como elementos necesarios para los avances heurísticos de la disciplina, excepto, por supuesto, que se adopte una perspectiva particular al respecto, como, por ejemplo, la investigación-acción participativa o la psicología crítica.

En suma, si nuestro propósito es reflexionar sobre los retos que nos esperan, conviene reconocer de entrada la diversidad de la psicología como disciplina y la relativa independencia de los desafíos que le aguardan a la profesión en lo sucesivo. Por supuesto, ello no implica que renunciemos a la pregunta sobre la coherencia (en un sentido que aclararé más adelante) de las teorías, los modelos y los métodos. Después de todo, hacemos ciencia en tanto que resolvemos nuestras diferencias mediante la argumentación pública, en el entendido de que no todas las posturas son igualmente válidas. En este sentido, podemos transigir con que la psicología sea diversa, pero no con que sea ecuménica. 


\section{Retos en el trasfondo de logros}

Asumiendo la diversidad de la psicología, pero no así su ecumenismo, quisiera entonces avanzar hacia la identificación de algunos desafíos que son al tiempo generales y relevantes para un conjunto importante de sub-áreas psicológicas. Una manera de identificarlos es percibirlos en el trasfondo de los logros de la disciplina o, cuando menos, de aquellos problemas que podemos considerar saldados en gran medida.

Debemos admitir que a pesar del carácter debatible de casi todo lo que se propone en psicología, nuestra disciplina ha hecho avances importantes. Estamos lejos de los sencillos modelos de frustración-agresión propuestos por Dollard y Miller en la década de los cuarenta para dar cuenta de los orígenes del comportamiento violento. Hoy sabemos, por ejemplo, que tanto la agresión reactiva como la proactiva son acciones simbólicas, en el sentido de que le permiten al agresor afianzar elementos de identidad en el contexto de las prácticas culturales en las que participa (Estrada, et al., 2005). James introdujo en sus Principles of Psycho$\log y$, la distinción entre memoria primaria y secundaria, pero hoy disponemos de modelos muy aproximados acerca del funcionamiento de la memoria de trabajo y de la representación del conocimiento en memoria a largo plazo. De hecho, las arquitecturas cognitivas actuales se han vuelto cada vez más consistentes con lo que hemos aprendido sobre el funcionamiento del cerebro (como ejemplo, ver Taatgen \& Anderson, 2009). Ha corrido mucha agua bajo el puente desde las concepciones clasistas de Terman sobre la inteligencia (Gould, 1981). Hoy reconocemos la influencia del conocimiento de dominio específico en la acción inteligente, al igual que su variabilidad sociocultural, y estamos, desde luego, mucho menos dispuestos a refrendar la reificación de la inteligencia (a manera de ejemplo, ver Cianciolo \& Stenberg, 2004). Por su parte, el desarrollo cognitivo ya no es concebido exclusivamente como una aventura epistemológica, sino como un proceso genuinamente entreverado en las interacciones sociales de las que es partícipe el niño (como ejemplo, ver: Rogoff, 2003; Tomasello, 1999).

En este tenor de cosas, existen entonces derroteros que han dejado de ser idearios para convertirse ya en lugares comunes de la disciplina. No sería razonable, por ejemplo, retar públicamente a la psicología, como en su momento lo hizo Watson en estilo de manifiesto, a recurrir a los fenómenos observables, es decir, a lo susceptible de escrutinio público, como fuente de la indagación psicológica. Creo no equivocarme al señalar que existe consenso respecto a la necesidad de modalidades más o menos laxas de conductismo metodológico, se trate de apuntalar inferencias conceptuales en conductas observables, tiempos de reacción, discurso, protocolos verbales, capturas de interacción social o medicio- 
nes de estados fisiológicos. El empleo de evidencia pública escrutable (incluso en forma de escritura etnográfica) no puede ser, entonces, un reto, porque es ya una condición de nuestra disciplina. Desde luego, la adhesión a versiones relajadas del conductismo metodológico no tiene por qué excluir posiciones críticas respecto a la supuesta transparencia de los protocolos observacionales.

Tampoco sería un reto especialmente original la adopción obligada de protocolos experimentales, bien porque en muchos campos de la psicología el experimento es el procedimiento estándar, bien porque existe comprensión (aunque infortunadamente parcial) respecto a las limitaciones de su uso. Al respecto, estamos cerca de una posición semejante a la de la física, que no reifica la cultura experimental como único acceso al conocimiento científico.

Otro tanto podría decirse del uso en la investigación psicológica de los análisis cualitativos, en contraposición con el análisis cuantitativo. Ambas estrategias de análisis han hecho parte de tradiciones muy respetadas en la psicología (Jacob, 1987). La cuantificación, en el ámbito de la evaluación y la medición de la inteligencia y de otros rasgos psicológicos, en los modelos matemáticos de aprendizaje, en las teorías probabilísticas de toma de decisiones y razonamiento moral, en las teorías estocásticas de formación de conceptos, y en la estimación de diferencias entre condiciones experimentales. Por su parte, los análisis cualitativos han jugado un papel central en la psicología clínica, la psicología ecológica, la psicología comunitaria, ciertas versiones de la psicología de la memoria (recuérdese a Frederic Bartlett), la psicología educativa y, más recientemente, la psicología crítica y discursiva.

Sin embargo, como señalaba Pintrich (2000), el debate entre lo cualitativo y lo cuantitativo, entendido como una oposición dicotómica, es un debate agotado, que recurre, no obstante, en contextos en los que suele desconocerse que la cuantificación debe ser entendida menos como una posición sobre el estatus ontológico de los rasgos psicológicos, que como una estrategia de reducción de datos para ciertos propósitos. En tal sentido, los análisis cuantitativos y cualitativos no tienen por qué oponerse, sino, por el contrario, integrarse, cuando co-ocurren, en el seno de un argumento más amplio. ${ }^{2}$

2 La posición que presento es, claramente, una posición pragmática que asume que, dadas ciertas preguntas, es posible integrar análisis cualitativos y cuantitativos en el contexto de argumentos más amplios. Sin embargo, esta posición no desconoce necesariamente que, como sugiere Shweder (1997), haya preguntas u objetos de estudio cualitativos, diferentes de las preguntas y los objetos de estudio cuantitativos. De hecho, la tensión entre los métodos cualitativos y cuantitativos, a pesar de haberse declarado difunta a finales de la década de 1990, ha cobrado, sin embargo, renovado vigor por efecto de cambios en las políticas de financiación a la investigación científica (particularmente, en campos como la salud y la educación), que ahora establecen los diseños experimentales aleatorizados como regla de oro para la conducción de investigación válida. En el campo cualitativo, desde luego, las respuestas no se han hecho esperar (ver: Packer, 2010). 
Mucho menos consensual, aunque en cierta medida agotado, es el debate sobre el lugar de la psicología en el seno de las ciencias naturales o las ciencias sociales o humanas. Es, sin duda, un asunto que ocupó un lugar privilegiado en las reflexiones epistemológicas de los psicólogos sobre su propia disciplina, particularmente en los años setenta, cuando menos en Latinoamérica (Larreamendy, et al., 2006). ${ }^{3}$ En Kinds of minds, Dennett afirmaba que si "el libre albedrío significa tanto para nosotros, debe ser porque no tenerlo sería terrible y porque puede haber razones para dudar de su existencia" (Dennett, 1992 : p. 18). Considerando la vehemencia del debate, supongo que lo mismo podría decirse de la significación de la ciencia para la psicología: si la ciencia significa y ha significado tanto para la psicología, es probablemente porque no tenerla, no ser una disciplina científica, sería algo terrible, o cuando menos implicaría la renuncia a metas que son consideradas caras, tales como la construcción de modelos y teorías, y el debate empírico alrededor de explicaciones (además de otras aspiraciones, más ligadas a versiones particulares de la ciencia, como es el caso de las proposiciones universales y las leyes sustantivas).

Sin embargo, el debate sobre si la psicología es una ciencia es, en mi opinión, un debate agotado, cuando menos en su versión original, no porque la respuesta sea obvia, sino porque la ubicación de la psicología en el concierto de las ciencias es un problema atravesado por la diversidad misma de la psicología y proclive a ser "resuelto" mediante una cuidadosa consideración de las características de la investigación empírica psicológica, más que por un consenso filosófico acerca de la bondad de ciertos desiderata epistemológicos. Dudo mucho que algún psicólogo pueda sensatamente negar que somos una disciplina científica, en el sentido específico de ser una comunidad generadora de conocimiento que está sometida al escrutinio público, que substancia sus enunciados teóricos con base enunciados empíricos y que adopta criterios, dependientes del contexto, para la evaluación de la validez de sus proposiciones.

Pero si hemos perdido interés en asuntos como los mencionados, ¿qué, entonces, debería convocar nuestra atención? Quisiera en lo sucesivo referirme a algunos desafíos que considero tienen relevancia para un muy amplio número de dominios dentro de la psicología.

\section{Proceso e historia}

El primero de ellos se refiere a la necesidad de desarrollar explicaciones que vayan más allá de la identificación de variables o factores que co-ocurren

3 El debate ha resurgido tímidamente en los Estados Unidos en el contexto de la polémica sobre los estándares de investigación científica en educación y salud (Penuel \& O’Connor, 2010). 
estadísticamente con el fenómeno. Dicho de manera simple, la psicología debe avanzar hacia modalidades explicativas más comprometedoras que el análisis de co-ocurrencia, el modelamiento estadístico y el análisis de datos agregados (Ohlsson, 2007).

La naturaleza de la explicación científica ha sido un tópico de intensa investigación en la filosofía y la historia de la ciencia durante cerca de cinco décadas (ver Salmon, 1989, para referencias). La magnitud y amplitud de los debates académicos, además de la creciente diversidad de las prácticas científicas, precluyen cualquier esperanza de proponer una definición taxativa y consensual de explicación científica. Sin embargo, existen algunos modelos clásicos sobre explicación que quisiera mencionar brevemente como coordenadas para la discusión. La perspectiva más ortodoxa e influyente sobre la explicación en ciencia ha sido el modelo deductivo-nomológico propuesto por Hempel y Oppenheim (1948). Según este modelo, las explicaciones son argumentos deductivos válidos en los cuales el evento a explicar se sigue, por un lado, de premisas derivadas de leyes generales, y por el otro, de una serie de condiciones iniciales. Así, por ejemplo, los físicos explican el color azul del cielo, derivando su ocurrencia de leyes generales acerca de la reflexión y absorción de las ondas de luz, e información específica sobre la composición física de la atmósfera terrestre.

En el modelo deductivo-nomológico, la explicación y la predicción están estrechamente ligadas. Un marco deductivo nos permite inferir a partir de las condiciones iniciales, con la ayuda de leyes generales, la ocurrencia de estados futuros. Al mismo tiempo, nos permite razonar de los fenómenos observados a las leyes o condiciones iniciales. En tal sentido, la predicción no se limita a enunciar la ocurrencia de un fenómeno, sino que implica ella misma un proceso derivativo preciso. Otro aspecto igualmente importante del modelo deductivonomológico es la distinción entre leyes explicativas y enunciados descriptivos (que pueden, ellos mismos, poseer el estatuto de leyes empíricas).

Una perspectiva alternativa al modelo deductivo-nomológico es el modelo estadístico. Según este modelo, una explicación puede definirse como un argumento en el que un evento es explicado si puede esperarse en virtud de la presencia ciertos hechos explicativos (Salmon, 1989). Explicar equivale entonces a identificar factores que son estadísticamente relevantes a la ocurrencia del explanandum. A pesar de la importancia de esta perspectiva, el modelo estadístico deja sin especificar los mecanismos mediante los cuales el evento está causalmente conectado a eventos previos o concurrentes, aunque la relación causal, como tal, se predique. 
El énfasis en las relaciones causales constituye, de hecho, el centro de una tercera perspectiva sobre las explicaciones científicas: el modelo causal. Según Salmon (1989 : p. 125), "una explicación científica de un evento o fenómeno consiste en citar una porción de los procesos causales y las interacciones conducentes a dicho fenómeno o evento". Ello implica reconstruir la cadena de estados conducentes a un evento y explicitar los mecanismos que permiten la transición de un estado a otro. Desde luego, no hay ningún compromiso de este modelo con cadenas causales lineales, ni exclusión de ámbitos, como los sistemas complejos, que son ontológica y no solamente epistemológicamente probabilísticos. En el modelo causal, el criterio más importante para evaluar la adecuación de la explicaciones es el grado de especificación de los mecanismos causales y de los factores relevantes al fenómeno a ser explicado.

La literatura psicológica, y en especial la literatura experimental, es particularmente abundante en relaciones estadísticas. A pesar de la popularización de los análisis cualitativos, aún ciframos enormes esperanzas en los artificios estadísticos que nos permiten establecer si dos o más grupos son diferentes, la incidencia de ciertos factores en la variabilidad del fenómeno estudiado y el tamaño del efecto encontrado. La estadística, mucho de cuyo desarrollo se debe precisamente a psicólogos, nos ha dotado de herramientas para predecir, diferenciar, agrupar y colapsar datos, muchas veces a expensas de nuestra habilidad para proponer explicaciones, por un lado, plausibles y por el otro, fundamentadas. Dicho de otra manera, la sofisticación de nuestros análisis estadísticos es contrastante con la pobreza y el simplismo de nuestras explicaciones.

Sabemos, por ejemplo, que los recuerdos autobiográficos en personas adultas (entre 60 y 65 años) presentan un pico entre las edades de 15 y 25 años, pero desconocemos por qué (Conway \& Pleydell Pearce, 2000). Según Perry Klein (2000) sabemos que escribir favorece el aprendizaje de dominios específicos de conocimiento, pero nuestros modelos acerca de cómo los procesos cognitivos implicados en la escritura potencian la comprensión son aún precarios. Sabemos de la robustez estadística de los efectos de recencia y primacía, pero desconocemos sobre los mecanismos cognitivos responsables de tales efectos. Existen modelos, basados en sofisticados análisis de regresión y ecuaciones estructurales, que han determinado el peso de factores (como la utilidad, la probabilidad de riesgo y otros) en el razonamiento moral. Pero carecemos de modelos que especifiquen cómo, a partir del conocimiento de los sujetos y de su experiencia previa, se identifican las funciones de utilidad y las probabilidades de riesgo.

Hemos sido disciplinariamente prolíficos en establecer relaciones y en muchos casos en realizar predicciones precisas. Hemos sido también cautos en no confun- 
dir las relaciones estadísticas con la causación. Sin embargo, aún tenemos mucho camino por recorrer en lo relativo a justificar la plausibilidad y los mecanismos generadores de los fenómenos o procesos que exitosamente predecimos, al igual que en aportar evidencia o diseńar condiciones de prueba de nuestras conjeturas explicativas causales, y no solamente de nuestras predicciones sobre relaciones. En términos de Herbert Simon, debemos avanzar hacia la postulación de teorías explicativas, basadas en modelos de proceso y no solamente en modelos de efecto.

Un ejemplo del desarrollo de explicaciones causales, cuya descripción de procesos va más allá del tratamiento estadístico, es la investigación actual sobre el efecto de tutoría sobre el aprendizaje de habilidades. En 1984, Benjamin Bloom publicó un artículo ya clásico en psicología educativa, titulado "El problema del 2-sigma: la búsqueda de métodos de enseñanza grupal tan efectivos como la tutoría uno-a-uno”. En este artículo, Bloom reportaba una serie de estudios comparativos entre situaciones de tutoría individual y enseñanza grupal en los que la diferencia entre una y otra condición, en términos de ganancias de aprendizaje, era del orden de dos desviaciones estándar. El reto que impuso Bloom en su momento fue explicar por qué. Desde entonces se han formulado diversas hipótesis para dar cuenta de las ganancias sustanciales que se obtienen mediante enseñanza tutorial. Algunas de ellas son el nivel de detalle de las evaluaciones diagnósticas, la selección individualizada de tareas, la sofisticación de las estrategias tutoriales, el control de los diálogos por parte del aprendiz, el conocimiento de dominio específico, la motivación, la auto-corrección de errores, el andamiaje [scaffolding] y el nivel de interactividad. Puesto que la modalidad de tutoría (i.e., lo que el tutor hace con el aprendiz) es dependiente del desempeño del aprendiz (i.e., el tutor termina haciendo lo que las condiciones del aprendiz le permiten), la asignación aleatoria de participantes a condiciones experimentales que representen las hipótesis anteriores es problemática y, por tanto, la conducción de experimentos en sentido estricto (Ohlsson, 2007). En su reemplazo, los investigadores han recurrido a los diseños cuasi-experimentales, llevando a cabo análisis estadísticos que regresan el desempeño de los estudiantes a ciertas características de la interacción (e.g., número de turnos conversacionales como indicador de interactividad).

Sin embargo, los estudios no se han detenido en la evidencia estadística (cada vez más consistente con la hipótesis de la interactividad). Investigadores como Kurt VanLehn y su grupo han desarrollado programas de inteligencia artificial (tutores inteligentes) que implementan adaptativamente una u otra estrategia de enseñanza tutorial de cara al desempeño del estudiante y que permiten observar y predecir, a nivel microgenético, las respuestas y ganancias cognitivas de los 
aprendices. La construcción de tutores inteligentes y el análisis microgenético del desempeńo de los estudiantes han permitido responder a la pregunta de si condiciones particulares favorecen el aprendizaje, pero también desarrollar modelos de cómo situaciones específicas de enseñanza permiten el desarrollo de habilidades cognitivas complejas (Rosé, et al., 2003; VanLehn, et al., 2003). La respuesta a ese cómo exige una perspectiva de proceso. Las explicaciones causales, es decir, las explicaciones que proporcionan un recuento explícito de las condiciones de producción de un fenómeno, son escasas, pero por lo menos existentes en la psicología, especialmente en terrenos como la psicología de la comprensión de textos, la producción de lenguaje, la resolución de problemas y el razonamiento.

Una alternativa a la especificación de procesos responsables por fenómenos de interés (especificación que tiene, sin duda, un sabor computacional) es la explicación por vías de la narración de las condiciones y dimensiones sociohistóricas que están implicadas en un fenómeno particular. Tómese, a manera de ejemplo, el caso del estrés laboral. Uno de los principales modelos para explicar el estrés laboral es el propuesto por Siegrist (1996), denominado Desbalance Esfuerzo-Recompensas, según el cual las situaciones estresantes son aquellas que se caracterizan por un alto esfuerzo y una baja recompensa. La situación laboral desfavorable surge, pues, de una percepción del desbalance entre esfuerzos realizados y recompensas recibidas. Se trata de un modelo que ha sido validado, fundamentalmente, a partir de la administración de instrumentos psicométricos que evalúan percepciones de esfuerzo y recompensa en ambientes laborales. Y, como cabría esperar, las relaciones estadísticas entre el desbalance de las mismas y la presencia de estrés han sido consistentemente significativas.

Sin embargo, enunciada en esos términos, la explicación sub-especifica las condiciones de posibilidad del fenómeno, al no aportar información alguna sobre las dimensiones históricas, culturales y personales que están implicadas en la significación o interpretación de una situación como esfuerzo o recompensa. Así, la parsimonia del modelo de Siegrist termina trivializándolo, es decir, volviendo indeseablemente poderosas sus explicaciones, al tiempo que poco específicas. Una solución a la débil especificación explicativa es la introducción de determinantes históricos, que permitan definir, tanto en el plano personal como en el cultural, los recursos a partir de los cuales categorías como las de recompensa y esfuerzo se construyen. Se dirá que, con todo, el modelo de Siegrist aspira a la simplicidad propia de la ciencia positiva, pero de lo que se trata con las explicaciones históricas es, como señalaba Vygotsky (1929/1889) parafraseando a Marx, elevar la explicación a lo concreto. 
Ciertamente no es el caso de la psicología avanzar hacia explicaciones deductivas, pues existen limitaciones poderosas a las explicaciones de este tipo en el caso de sistemas fenoménicos en los que las condiciones contingentes y muchas veces azarosas generan efectos considerables e impredecibles - para no mencionar el hecho, quizás aún más primario, de que disponemos de muy pocas leyes generales. Pero sí considero, de la mano de Newell y Simon (1972), que es necesario diseñar modelos y teorías de proceso cuya validez no proceda exclusivamente de un tratamiento estadístico de datos agregados. Dicho en otras palabras, podríamos avanzar hacia la consolidación de una psicología genuinamente explicativa, concediéndole al modelo epidemiológico su justo lugar. Ello implica, según sea del caso, arriesgar explicaciones causales de proceso o avanzar hacia explicaciones densas, desde el punto de vista histórico, cultural y ontogenético.

\section{Coherencia explicativa}

Avanzar hacia una más estrecha relación entre las explicaciones psicológicas y las explicaciones de otros dominios disciplinares es el segundo desafío que desearía proponer. En más ocasiones de las que quisiéramos, la psicología ha sido una disciplina vergonzante, culposamente superficial respecto a la filosofía, miope respecto a la sociología, si comparada con la fisiología, alquimista en relación con la física y estéril al lado de la literatura. Al respecto cabe recordar el placer con que algunos de nuestros colegas señalan cómo muchas de nuestras grandes figuras no fueron psicólogos de formación.

Esa relación problemática con otras disciplinas ha dado origen, si se permite la licencia, a un cierto provincialismo disciplinar. A manera de ejemplo, la psicología ha postulado mecanismos sobre la adquisición de la lengua sin consultar la naturaleza del lenguaje (como ocurrió con la propuesta de Skinner en Verbal Behavior); y ha formulado teorías sobre la inteligencia que entran en franca contradicción con la biología y con la función de la inteligencia, como rasgo adaptativo, en la evolución de la especie, como es el caso de las teorías de dominio general. A esta tendencia la conocemos con el nombre de psicologismo, que no es otra cosa que suponer que el plano de la explicación psicológica no sólo es diferenciable de otros planos explicativos (que lo es legítimamente), sino también autónomo (que, por supuesto, no lo es).

En ańos recientes, sin embargo, se observa un mayor cuidado en proponer explicaciones psicológicas que son, no solamente consistentes con otros desarrollos en la psicología misma, sino también coherentes con marcos explicativos consolidados en otras disciplinas. Por ejemplo, algunos desarrollos recientes sobre el concepto de competencia laboral en el ámbito organizacional están en creciente 
correspondencia con los avances en psicología cognitiva y teoría sociocultural sobre la naturaleza del conocimiento y el aprendizaje (Brown \& Duguid, 1991; Lave \& Wenger, 1991; Orr, 1990). Son más interesantes, empero, los vínculos interdisciplinarios. Un ejemplo notable, aunque desde luego no carente de críticas, es la psicología evolucionaria (ver: Buss, 2009a, 2009b, para referencias). La psicología evolucionaria se sirve del esquema amplio de la síntesis neo-darwiniana para proponer explicaciones genuinamente psicológicas que son, no obstante, consistentes con nuestro conocimiento actual de la evolución de la especie. El naciente campo de la psicología evolucionaria -que no debe confundirse con la psicología evolutiva de corte piagetiano-, ha explicado, con base en su valor adaptativo, mecanismos psicológicos implicados en la preferencia de ciertas comidas, el origen de los celos masculinos, las preferencias en la selección de parejas, los procedimientos de detección de engaño, la adquisición del lenguaje, y los procesos de disolución marital. Como puede verse, no se trata de hacer biología donde tendría que hacerse psicología, sino de hacer una psicología biológicamente plausible.

Otro tanto sucede en el campo de la psicología cognitiva. Aunque las explicaciones basadas en arquitecturas simbólicas han sido enormemente productivas, la necesidad de conciliar las explicaciones cognitivas con las neurociencias ha resultado determinante en la promoción de investigaciones sobre conexionismo y arquitecturas basadas en procesamiento paralelo, que guardan una mayor correspondencia con lo que sabemos actualmente sobre el cerebro (Taatgen $\&$ Anderson, 2009). De igual manera, en la actualidad las teorías sobre adquisición de la lengua materna postulan mecanismos que son consistentes con aspectos formales del lenguaje ya de sobra conocidos, con restricciones de procesamiento ampliamente documentadas (como es el caso del encapsulamiento sintáctico) y con lo que podemos sensatamente suponer fue la evolución del lenguaje en la especie. En tal sentido, la psicología está llamada en los próximos años a realizar avances significativos en el área de las neurociencias, que de suyo presuponen una coherencia explicativa obligada. El reto es, en mi opinión, lograr una coherencia explicativa sin derivar en un fisicalismo o un eliminacionismo a ultranza.

Como puede colegirse, estoy abogando, no por el resurgimiento de las grandes teorías, bajo cuya sombra se formaron generaciones de psicólogos. No se trata de resucitar o avanzar hacia sistemas doctrinarios que impongan una concepción del mundo basada en principios ontológicos, explicativos y metodológicos excluyentes, de dominio general. De hecho, si algo tenemos que poder lograr es hacer gravitar nuestra investigación alrededor de problemas, sobre los cuales 
pueda pronunciarse una amplia y diversa audiencia, sin que estemos sometidos a la lógica excluyente, pero también complaciente, de los enfoques.

El derrotero es, si se quiere, mucho más modesto, pero también más exigente. Se trata de preguntarnos incesantemente por la coherencia empírica y semántica entre nuestras explicaciones y las explicaciones alternativas que se formulan en otras psicologías sobre los mismos fenómenos. Pero también se trata de hacer nuestras explicaciones psicológicas compatibles, en algún sentido significativo, con los principios físicos, biológicos y culturales, que suponemos funcionan como dimensiones, restricciones o posibilitantes [constraints y affordances] del suceso psicológico.

Dicho en pocas palabras, creo que debe hacer parte de la agenda psicológica la exigencia de una coherencia explicativa entre lo que propone la psicología, como disciplina, y otras ciencias, cuya validez estamos dispuestos a aceptar. Entre ellas, supongo que serán de especial importancia las neurociencias y la biología.

\section{Cotidianidad}

Existen, desde luego, desafíos en terrenos no tan áridos como los mencionados hasta ahora. Quisiera mencionar dos que me parecen de especial importancia. El primero es lo que llamaría la incursión en lo cotidiano. Obviamente, la psicología siempre ha tenido que ver con las vidas de las personas; es decir, con ese orden de la experiencia al que denominamos vida cotidiana. ${ }^{4}$ Después de todo, eso es lo que se ventila en una psicoterapia (Dreier, 2008). Es igualmente cotidianidad la vida de una comunidad, la relación de una pareja y el desarrollo socioemocional de un niño, para nombrar tan sólo algunos ejemplos. Pero quisiera corregir. La psicología no siempre ha tenido que ver con la vida cotidiana. La cultura experimental en psicología ha abstraído los grupos de sus encuadres culturales y organizacionales; el aprendizaje, de la socialización, la formación de identidad y el trabajo; la inteligencia, de la resolución de problemas adaptativos y significativos; la familia, de la cultura que recrea; la agresión, de la dinámica de identidades; y la violencia, de su contexto sociopolítico y subjetivo.

Las críticas a la lógica del laboratorio datan de décadas atrás, al igual que la introducción de conceptos como el de validez ecológica. No es un problema nuevo. Sin embargo, y a pesar de las concesiones, la distinción entre la investigación básica y la investigación aplicada ha permanecido intacta en la mentalidad de muchos, en parte como efecto de una división del trabajo en la comunidad

4 Esto, desde luego, a pesar de que la inmensa mayoría de la investigación psicológica experimental, que ha sido la predominante en buena parte de las áreas disciplinares, haya sido conducida en condiciones de dudosa autenticidad. 
psicológica, en parte como consecuencia de la muy discutida creencia de que la complejidad de escenarios de investigación obscurece la identificación de principios fundamentales. Se trata, como puede verse, de una posición diametralmente opuesta a la perspectiva adoptada por otras ciencias sociales (como la antropología), en las cuales la etnografía holística ha cumplido las veces del experimento en nuestra disciplina.

Sin embargo, hay voces disidentes. Por ejemplo, Ed Hutchins (1995) ha venido estudiando desde hace más de dos décadas procesos de resolución de problemas y comprensión cognitiva en ambientes enteramente cotidianos: desde la navegación marítima sin instrumentos de aborígenes polinesios, hasta los procesos de memoria que ocurren en la cabina de un jet comercial. Sylvia Scribner (1985), una figura mayor en el desarrollo de la psicología cultural, investigó en su momento la manera como los trabajadores de una procesadora y distribuidora de lácteos resolvían problemas que implicaban, en principio, complejos razonamientos matemáticos. Gaea Leinhardt y colegas (Leinhardt, et al., 2002) han investigado la manera como las personas aprenden ciencia en museos y contextos informales. John Brown y Paul Duguid (1991) han intentado atacar el problema de cómo la gente aprende en los contextos de trabajo, en sus ocupaciones, cerrando así la brecha entre aprendizaje y trabajo.

Las anteriores son referencias que me son familiares porque en algún sentido interceptan la cognición y la educación. Sin embargo, hay en dichas experiencias algo que es común a muchos otros dominios de la psicología. Supongo que ninguno de los investigadores citados admitiría de buena gana que su indagación no tiene que ver con los problemas fundamentales de la psicología. Por ejemplo, investigar cómo los miembros de una organización aprenden al margen de estrategias formales de enseñanza nos dice algo fundamental acerca del aprendizaje en la vida humana; algo que no es menos fundamental que lo que un analista del comportamiento o un psicólogo cognitivo podría decirnos a partir de cuidadosos estudios de laboratorio. De la misma manera, cuando Hutchins investiga cómo los tripulantes de un barco colaboran para llevar la nave a aguas seguras, hay allí algo que escapa a la lógica de la aplicación de principios fundamentales y que aporta elementos nuevos a nuestra comprensión de la naturaleza del aprendizaje. Podría decirse lo mismo sobre las luces que proporciona un estudio sobre la resolución de problemas en culinaria a la comprensión de los mecanismos de la inteligencia humana. Algunas de estas referencias pueden, sin duda, parecer exóticas, pero la vida cotidiana es harto exótica, cuando la medimos con el rasero de la asepsia experimental. 
Los estudios citados presumen que no es posible explicar los hechos psicológicos al margen de las actividades, los contextos socioculturales y los motivos de los cuales hacen parte. En tal sentido, ponen en duda la plausibilidad de estudiar, digamos, el razonamiento moral, al margen de los propósitos que alientan los mecanismos de razonamiento, del ethos que actúa como justificación, o de los contenidos sobre los cuales el razonamiento moral opera; ponen en duda la posibilidad de hablar de una lógica de las organizaciones que desconozca los procesos de socialización típicos de una cultura; ponen en duda la posibilidad de hablar de motivación, como si se tratase de una substancia, al margen de las coordenadas subjetivas que hacen consistir nuestros deseos; ponen en duda la posibilidad de estudiar la inteligencia con independencia de los problemas adaptativos que resuelve y que, por tanto, son significativos para el sujeto; ponen en duda la posibilidad de hablar del desarrollo al margen de la educación y de la identidad al margen de los procesos sociales específicos de reconocimiento y discriminación.

Visto así, el viraje hacia lo cotidiano reside en hacer una psicología no tanto parroquial, o vacía de enunciados generales, como informada por la particularidad de los contextos en los cuales ocurren los hechos psicológicos de interés. Esos contextos, lo sabemos, son considerablemente más ruidosos de lo que desearíamos; pero restituyen la complejidad real de los fenómenos. No estudiamos al ser humano en general, aunque al cabo podamos decir algo razonable acerca de la especie. Estudiamos seres particulares, cuyo contexto (físico, natural y social) moldea, restringe y posibilita de manera muy especial esos procesos en los que estamos a menudo interesados.

El análisis de situaciones cotidianas, enriquecidas, muchas de ellas irrepetibles, está llamado a aportar elementos fundamentales a la respuesta de los interrogantes básicos de la psicología. No creo que nuestra creciente proclividad por lo que he denominado cotidiano desdibuje las fronteras entre lo disciplinario y lo profesional, pero sí puede restituir el valor heurístico de las situaciones a las que el profesional está habituado, de cara al avance del conocimiento psicológico. Adicionalmente, puede contribuir a acentuar el papel social de la disciplina psicológica como la encargada de generar conocimiento sobre lo que somos, no el ser humano en general, sino nosotros.

\section{Qualia y experiencia}

De la mano con la cotidianidad, uno de los retos que espera a la psicología es el que se le propuso en el momento mismo de su fundación como disciplina independiente; a saber, la explicación y comprensión del fenómeno de la concien- 
cia. Como sabemos, la conciencia, como objeto de estudio, fue un tema relegado por mucho tiempo, porque era y continúa siendo particularmente refractario a la evidencia pública. Sin embargo, como señalaba William James, la conciencia es un dato primario, desde luego, no porque no pueda ser analizado, sino porque hace parte fundamental de la experiencia humana.

El problema de la conciencia es, sin duda, un problema escurridizo, sobre el cual poco tienen que decir las tradiciones ortodoxas del análisis experimental del comportamiento y las versiones más positivistas de la psicología cognitiva. Recuérdese que Thomas Nagel, en su célebre artículo "What is it like to be a bat?" (1974), lo elevó al estatuto de límite de la psicología (cognitiva). Es también un problema que dio origen a la clásica distinción de Dilthey entre explicación y comprensión. Y es un problema que podría, no obstante, con base en un purismo metodológico, relegarse al cuarto de San Alejo, de no ser, repito, porque constituye un aspecto esencial de nuestra experiencia y porque ha comenzado a ser investigado la originalidad que merece.

De hecho, el problema de la consciencia, cuando menos en una de sus aristas, guarda una estrecha relación con lo que en filosofía se ha dado en denominar qualia, un término referido al carácter fenoménico de una experiencia, es decir, a lo que se "siente" vivir una experiencia particular. Recientemente, Eleanor Maguire y colaboradores (Hassabis, et al., 2009), de la University College en Londres, lograron predecir, a partir de fMRI de la actividad del lóbulo temporal medio de personas durante una tarea de navegación en un ambiente de realidad virtual, la "posición" de un individuo dentro de dicho ambiente. Como cabría esperar, este hallazgo ha suscitado un enorme interés, porque es una primera evidencia de que representaciones espaciales complejas están asociadas a patrones de activación específicas. Pero, desde luego, lo que la visualización a partir de fMRI no proporciona (y no pretende hacerlo) es evidencia acerca de qué "se siente" tener un pensamiento particular, o, como diría James, ser parte de una particular corriente de pensamiento [stream of thought].

Además de su interés inherente, el problema de la conciencia reviste de especial importancia porque a su elusividad no puede sino responderse con creatividad metodológica. De hecho, son los problemas que no son tratables con estrategias estándar aquellos que nos permiten avanzar en nuestros métodos de indagación. Por ejemplo, Anthony Jack y Tim Shallice (2000) han abogado por el empleo de evidencia introspectiva, desde luego en condiciones de validez y confiabilidad superiores a las que motivaron su abandono hace ya un siglo. Por su parte, Ricouer (1978) ha argumentado convincentemente que el abismo entre explicación y comprensión, que se origina en la intractabilidad de la con- 
ciencia, puede ser enfrentado a través del relato, de la narrativa. Tal ha sido, de hecho, la propuesta metodológica de muchas de las investigaciones de género, que recurren a la narrativa como una estrategia para convenir una experiencia que es de suyo inconmensurable.

De hecho, una de las consecuencias de tomarse en serio la qualia como reto investigativo es el posicionamiento de la metodología cualitativa en nuestra disciplina. La investigación cualitativa tiene ya asegurado un lugar en la psicología, quiero decir, más allá de toda duda. El debate persiste, sin embargo, en relación con las promesas de la investigación cualitativa, es decir, con aquello que podría aportar y que constituiría en últimas su singularidad (Denzin \& Lincoln, 2005; Packer, 2010). En esa dirección, Shweder señala que:

[...] la tensión entre lo cuantitativo y lo cualitativo gira menos alrededor de cuestiones metodológicas que alrededor de nuestras respuestas a la preguntas de cómo estudiar mejor la subjetividad y la realidad que es dependiente de la perspectiva y del contexto. Básicamente, es la diferencia entre estudiar algo que existe independientemente de las reacciones que alguien tenga sobre ello y el estudio de las cosas que llegan a existir en virtud de su significado y la perspectiva que se adopte sobre ellas (Shweder, 1997: 160).

De hecho, Shweder mismo ha definido la investigación cualitativa (y, en particular, la etnografía) como el estudio de la qualia, en el sentido del estudio de la experiencia vivida, de la experiencia ineludiblemente determinada por la perspectiva del individuo y la cultura. En tal sentido, el problema de la conciencia y de la qualia como experiencia tendrán una importancia crítica en la comprensión de fenómenos que hoy en día son puntos ciegos, no tanto profesional, como disciplinariamente, como es el caso del dolor y el sufrimiento, pero también del estrés, la angustia, la felicidad y eso que denominamos, de manera muy ingenieril, calidad de vida.

\section{A manera de conclusión}

Me he detenido en cuatro retos para la psicología que se me ocurren generales: primero, la necesidad de desarrollar explicaciones de proceso e históricas que, más allá de la constatación de efectos y de los análisis agregados, den cuenta de la determinación o causación de sucesos psicológicos de interés; segundo, la necesidad de hacer consistir nuestras explicaciones con las explicaciones y las regularidades empíricas de otras psicologías y disciplinas, al márgen de la pretensión de resucitar los enfoques como Weltanshuung o de buscar amparo en otras disciplinas; tercero, la necesidad de investigar la cotidianidad, o mejor, los sucesos 
psicológicos que han sido siempre importantes para la disciplina en contextos genuinos y enriquecidos; y cuarto, la necesidad de explicar o comprender, según se prefiera, la experiencia subjetiva.

Mirados en conjunto, estos cuatro desafíos nos podrían conducir a una disciplina más rigurosa, distinta de la psicología popular [folk psychology], no tanto en sus intuiciones como en su sofisticación explicativa; a una disciplina menos aislacionista y más integrada al conjunto de las ciencias biológicas y las ciencias humanas, independientemente de si la psicología se adscribe o no a tal o cual área de conocimiento; a una disciplina que tenga algo realmente importante que decir sobre la manera como actuamos en la vida cotidiana y sobre las experiencias que nos son únicas e inconmensurables. Es claro que existen otros muchos desafíos y es del todo probable que los que he señalado no sean los más significativos. En cualquier caso, la diversidad de la psicología, que en cierta manera hace honor a la diversidad humana, nos permite repetir, con esperanza, las palabras del contramaestre de La Tempestad de Shakespeare: " $Y$ ahora viento, sopla hasta que revientes, visto que tenemos sitio para maniobrar".

\section{Bilbiografía}

Becher, Tony \& Trowler, Paul R. (1989). Academic tribes and territories. Buckingham: The Society for Research into Higher Education and Open University Press.

Bloom, Benjamin S. (1984). "The 2 sigma problem: the search for methods of group instruction as effective as one-to-one tutoring." Educational Researcher, 13, 4-16.

Brown, John, \& Duguid, Paul (1991). Organizational learning and communities of practice: Toward a unified view of working, learning, and innovation. Organizational Science, 2 (1), 40-57.

Buss, David M. (2009a). The great struggles of life: Darwin and the emergence of evolutionary psychology." American Psychologist, 64, 140-148.

Buss, David M. (2009b). How can evolutionary psychology successfully explain personality and individual differences?", Perspectives on Psychological Science, 4, 359-366. 
Cianciolo, Anna T., \& Sternberg, Robert. J. (2004). Intelligence: A brief history. Malden, MA: Blackwell Publishing.

Conway, Martin A., \& Pleydell Pearce, Christopher W. (2000). "The construction of autobiographical memories in the self memory system". Psychological Review, 107, 261-288.

Dennett, Daniel C. (1996). Kinds of minds: toward an understanding of consciousness. New York: Basic Books/ Harper Collins.

Denzin, Norman K; \& Lincoln, Yvonna S. (2005). Introduction: The discipline and practice of qualitative research. En N. K. Denzin \& Y. S. Lincoln (Eds.), The Sage Handbook of Qualitative Research (3rd ed.), pp. 1-32. Thousand Oaks, CA: Sage.

Dreier, Ole (2008). Psychotherapy in everyday life. New York: Cambridge University Press.

Estrada, Angela María; Ibarra, Carolina; \& Sarmiento, Estefanía. (2005) Regulation and control of subjectivity and private life in the context of armed conflict in Colombia. Journal of Family, Community and Psychology, 15, 133-149.

Feynman, Richard (1970). The Feynman lectures on physics. New York: Addison Wesley.

Gould, Stephen Jay. (1981). The Mismeasure of man. New York: W.W. Norton $\&$ Co.

Green, Christopher. D. (2009). The curious rise and fall of experimental psychology. History of the Human Sciences, 22, 33-53.

Hassabis, Demis; Chu, Carlton; Rees, Geraint; Weiskopf, Nikolaus; Molyneux, Peter D.; \& Maguire, Eleanor A. (2009). Decoding neuronal ensembles in the human hippocampus. Current Biology, 19(7), 546-554.

Hempel, Carl \& Oppenheim, Paul (1948). Studies in the logic of explanation. Philosophy of Science, 15, 135-175. 
Hutchins, Edwin (1995). Cognition in the wild. Cambridge, MA: MIT Press. Ibañez, Tomás. \& Íñiguez-Rueda, Luis. (Eds). (1997). Critical social psychology. London: Sage.

Jack, Anthony I., \& Shallice, Tim (2001). Introspective physicalism as an approach to the science of consciousness. Cognition, 79 (1-2), 161-196.

Jacob, Evelyn. (1989). Qualitative research traditions: a review. Review of Educational Research, 57 (1), 1-50.

Klein, Perry D. (2000). Elementary students' strategies for writing-to-learn in science. Cognition and Instruction, 18, 317-348.

Larreamendy-Joerns, Jorge; Henao, Juanita; \& Arango, Alexandra. (2006). Emergencia de la investigación cualitativa en psicología en Colombia: Un comienzo que aún no termina. Forum: Qualitative Social Research, 7, Septiembre 2006.

Lave, Jean. \& Wenger, Etienne. (1991). Situated learning: Legitimate peripheral participation. New York: Cambridge University Press.

Leinhardt, Gaea., Crowley, Kevin., \& Knutson, Karen. (Eds.) (2002). Learning conversations in museums. Mahwah, NJ: Lawrence Erlbaum Associates.

Mayr, Ernst. (1982). The growth of biological thought: Diversity, evolution, and inheritance. Cambridge, MA: Belknap Pres

Nagel, Thomas. (1974). What is it like to be a bat? Philosophical Review, LXXXIII, 4, 435-450.

Newell, Allen \& Simon, Herbert (1972). Human problem solving. Englewood Cliffs, NJ: Prentice Hall.

Penuel, William R., \& O'Connor, Kevin (Eds.) (2010). Learning Research as a Human Science. National Society for the Study of Education Yearbook, 109(1).

Ohlsson, Stellan (2007). Psychology is about processes. Integrative Psychological and Behavioral Science, 41, 28-34 
Orr, Jane (1990). Talking about Machines: An ethnography of a modern job. Disertación doctoral no publicada. Cornell University, Department of Anthropology.

Packer, Martin (2010). The science of qualitative research: Toward a historical ontology. Cambridge, MA: Cambridge University Press.

Parker, Ian (1999). Critical psychology: critical links. Annual Review of Critical Psychology, 1, 3-18.

Pera, Marcello (1994). The discourses of science. Chicago, IL: Chicago University Press.

Pintrich, Paul (2000). Educational psychology at the millennium: a look back and a look forward. Educational Psychologist, 35 (4), 221-226.

Ricœur, Paul (1978). Explanation and understanding: On some remarkable connnections among the theory of text, theory of action and theory of history. En C. E. Reagan and D. Stewart (Eds.), The philosophy of Paul Ricoeur: An anthology of his work. Boston: Beacon Press.

Rogoff, Barbara (2003). The cultural nature of human development. New York: Oxford University Press.

Rosé, Carolyn. P., Bhembe, Dumiziswe; Siler, Sue., Srivastava, Ram., \& VanLehn, Kurt. (2003). The role of why questions in effective human tutoring. En H. U. Hoppe, F. Verdejo and J. Kay (Eds.), Artificial Intelligence in Education. Amsterdam: IOS Press.

Salmon, Wesley C. (1989). Four decades of scientific explanation. En P. Kitcher \& W. C. Salmon (Eds.), Scientific explanation, Minnesota studies in the philosphy of science, Vol XIII (pp. 3-219). Minneapolis, MN: University of Minnesota Press.

Sampson, Edward E. (1981). Cognitive psychology as ideology. American Psychologist, 36, (7), 730-743.

Sawyer, R. Keith. (Editor). (2006). Cambridge handbook of the learning sciences. New York: Cambridge University Press. 
Scribner, Sylvia. (1985). Knowledge at work. Anthropology \& Education Quarterly, 16 (3), 199-206.

Shweder, Richard. (1997). The surprise of ethnography. Ethos, 25(2), 152-163. Siegrist, Johannes. (1996). Adverse health effects of high-effort/low-reward conditions. Journal of Occupational Health Psychology, 1, 27-41.

Taatgen, Niels. A. \& Anderson, John R. (2009). The past, present, and future of cognitive architectures. Topics in Cognitive Science, 1-12.

Tomasello, Michael. (1999). The cultural origins of human cognition. Harvard University Press.

VanLehn, Kurt., Siler, Stephanie., Murray, R. Charles., Yamauchi, Takashi., \& Baggett, William. B. (2003). Why do only some events cause learning during human tutoring? Cognition and Instruction, 21(3), 209-249.

Vygotsky, Lev S. (1929/1989). Concrete human psychology. (Orig. 1929) Soviet Psychology, 27(2), 53-77.

Weinberg, Steven. (1993). Dreams of a final theory: the search for the fundamental laws of nature. London: Vintage. 
